\section{$\underset{\substack{\text { hommes } \\ \text { \& migrations }}}{ }$}

\section{Hommes \& migrations}

Revue française de référence sur les dynamiques

migratoires

\section{$1284 \mid 2010$}

Migrations et environnement

\title{
La Cité crée son prix
}

Le prix littéraire de la Porte Dorée

\section{Élisabeth Lesne}

\section{Q OpenEdition \\ 1 Journals}

\section{Édition électronique}

URL : http://journals.openedition.org/hommesmigrations/1267

DOI : 10.4000/hommesmigrations. 1267

ISSN : 2262-3353

Éditeur

Musée national de l'histoire de l'immigration

\section{Édition imprimée}

Date de publication : 1 mars 2010

Pagination : 174-179

ISSN : 1142-852X

\section{Référence électronique}

Élisabeth Lesne, «La Cité crée son prix », Hommes \& migrations [En ligne], 1284 | 2010, mis en ligne le 29 mai 2013, consulté le 24 septembre 2020. URL : http://journals.openedition.org/ hommesmigrations/1267; DOI : https://doi.org/10.4000/hommesmigrations.1267

Ce document a été généré automatiquement le 24 septembre 2020.

Tous droits réservés 


\title{
La Cité crée son prix
}

\author{
Le prix littéraire de la Porte Dorée
}

\section{Élisabeth Lesne}

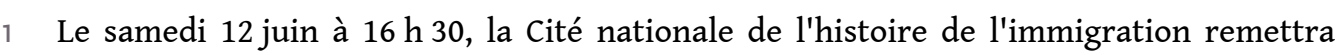
pour la première fois un prix à l'auteur d'un roman ayant pour thème l'exil. Cet événement aura lieu au Palais de la Porte Dorée ${ }^{1}$, en musique grâce à Julien Jacob et avec des lectures d'extraits par des comédiens, dans le cadre du festival "Paris en toutes lettres".

2 Le prix littéraire de la porte Dorée, doté de 4000 euros, doit son nom au Palais où est installée la Cité, mais c'est aussi un hommage à la Golden Door, le symbole des migrants voguant vers l'Amérique. Sur le socle de la statue de la liberté à New York, on peut lire ces vers d'Emma Lazarus :

"Send these, the homeless tempest tossed to me, I lift my lamp beside the golden door !"

"Envoie-les-moi, les déshérités, que la tempête me les apporte.De ma lumière, j’éclaire la porte d'or !"

\section{De Nouvelles Odyssées}

L'idée de ce prix est venue tout naturellement après l'édition par la Cité de Nouvelles Odyssées ${ }^{2}$, un recueil d'extraits d'écrivains racontant l'immigration. Conçu comme l'accompagnement littéraire de l'exposition permanente "Repères", ce recueil montre combien l'exil, qu'il soit volontaire ou imposé, économique ou politique, marque la littérature. Les souffrances et les angoisses, mais aussi les découvertes et parfois le sentiment de libération lié à l'éloignement de la terre natale sont depuis longtemps source de créativité et d'inspiration. Face à la richesse de ces œuvres, à leur intérêt littéraire et humain pour comprendre de l'intérieur, et non à coup de statistiques et de quotas, l'expérience de l'exil et de l'immigration, l'évidence s'est imposée : il fallait que la Cité crée un prix!

4 La mission de la médiathèque de la Cité étant de réunir et d'offrir au public tous les écrits, essais, récits, romans, ayant pour thème l'immigration, restait à faire un choix dans la production romanesque de l'année éditoriale 2009-2010. Cela aurait été difficile 
sans le comité de lecture composé de documentalistes, d'historiens, d'enseignants, de journalistes et de membres de la Cité, qui suit la constitution du fonds de la médiathèque Abdelmalek Sayad depuis le début.

\section{Comité de lecture et présélection}

5 Plus de 60 romans et récits écrits en français ont été lus, analysés, commentés, défendus ou exclus au cours de six séances passionnées, car les avis étaient loin d'être toujours convergents. L'objectif était de sélectionner une dizaine de titres avant le 8 mars et d'en remettre la liste à un jury pour la sélection finale du lauréat.

Preuve que le thème de l'exil intéresse et inspire, 3 titres qui nous ont séduits dès septembre ont reçu trois des grands prix littéraires de novembre: Trois femmes puissantes de Marie Ndiaye (prix Goncourt), L'Enigme du retour de Dany Laferrière (prix Médicis) et Le Club des Incorrigibles Optimistes de Jean-Michel Guenassia (prix Goncourt des lycéens).

7 Nous avons donc, à regret, décidé de les ôter de notre sélection pour mettre en avant des titres un peu moins connus, auxquels nous souhaitons de nombreux lecteurs :

- L'Arabe d'Antoine Audouard,

- Le Tombeau de Tommy d'Alain Blottière,

- Murmures à Beyoglu de David Boratav,

- Missak de Didier Daeninckx,

- La Mer noire de Kéthévane Davrichewy,

- En direction du vent de Fawaz Hussain,

- Tâche de ne pas devenir folle de Vanessa Schneider,

- Ru de Kim Thúy

- Jusque dans nos bras d'Alice Zeniter.

\section{Échos}

On peut s'amuser à tisser quelques liens entre ces 10 titres. Par exemple, dans des romans d'un style très différent, Daniel Daeninckx et Alain Blottière ont tous les deux fait revivre les résistants FTP-MOI du groupe Manouchian exécutés le 21 février 1944, le premier en s'attachant à l'Arménien Missak Manouchian, le second en faisant revivre le juif hongrois Thomas Elek.

Si Kéthévane Davridrewy et Vanessa Schneider ont une approche plus intime, puisqu'elle passe par l'histoire de leurs grands-mères, l'une est géorgienne, l'autre roumaine, c'est pourtant tout le $\mathrm{xx}^{\mathrm{e}}$ siècle qui est revisité à travers ces récits de vies tumultueuses. Et il est touchant de voir quelles traces laisse le pays d'origine, deux générations plus tard.

Moins évident peut paraître le rapprochement entre Antoine Audouard et Alice Zeniter, mais ces deux écrivains nous parlent de racisme, l'un dans sa version la plus noire, en décrivant les troubles que provoque l'arrivée d'un émigré algérien dans un village, l'autre avec verve et humour en racontant les préparatifs d'un mariage blanc entre deux amis d'enfance.

11 Quant à David Boratav et Fawaz Hussain, leurs narrateurs ont perdu leur père et c'est pour eux l'occasion de retourner le premier en Turquie, le second en Syrie, et de 
s'interroger sur les multiples facettes de l'identité, sur “l'autre langue” aussi. D'un côté, nous avons un premier roman complexe et intense, de l'autre, une ballade touchante dans le Paris des émigrés solitaires. Kim Thúy nous offre le récit le plus autobiographique, mais cette Vietnamienne émigrée au Canada évoque également la richesse et les déchirements de ceux qui vivent entre deux cultures, entre deux mondes : celui qu'ils ont laissé en émigrant, et celui où ils sont arrivés et ont choisi de rester.

\section{Jury et lauréat} rubriques société, politique et faits divers des médias. Que vive le prix de la Porte Dorée!

\section{L'Arabe d'Antoine Audouard (L'Olivier)}

Dans un village du sud de la France débarque un jour un jeune Arabe, qui n'aura jamais de nom. On lui loue une cave donnant sur la place du village, et le jour, il charrie des tonnes de cailloux sur un chantier. Mais en moins de deux, rien ne lui sera épargné : d'abord soupçonné de complicité de meurtre, il sera dénoncé pour tentative de viol, après avoir été suspecté d'avoir des accointances avec les islamistes... Dans ce roman qui montre combien les préjugés racistes peuvent être ancrés et nocifs, rien de manichéen pourtant. L'écriture, mélange d'oralité et de classicisme, de vulgaire et d'élégance, rend ce récit oppressant, jamais complaisant ni malsain.

\section{Murmures à Beyoglu de David Boratav (Gallimard)}

En pleine crise de la cinquantaine, insomniaque, quitté par sa femme et de plus en plus étranger à son fils, le narrateur doit aller enterrer son père à Paris. Arrivé de Turquie en France à l'âge du collège, il préfère désormais vivre à Londres, mais c'est à Istanbul qu'il va devoir se rendre après l'enterrement. Dans cette ville qui le rend d'abord mutique, il revit des scènes de son enfance qui se mêlent à la découverte de la capitale moderne, entre Orient et Occident. Pour retrouver le sommeil et l'accès à ses rêves, il lui faudra, au prix d'un ébranlement avec lequel les secousses du tremblement de terre entrent en écho, redécouvrir d'où il vient. Ce premier roman analyse avec finesse et 
humour les déchirements de ceux qui vivent entre deux mondes, les multiples facettes de l'identité, ses rumeurs et ses murmures.

\section{Le Tombeau de Tommy d'Alain Blottière (Gallimard)}

17 Ce roman raconte en miroir l'histoire de Thomas Elek, jeune juif hongrois résistant FTP-MOI exécuté avec ceux de "l'Affiche rouge", et le tournage d'un film sur sa courte vie. C'est Gabriel, adolescent parisien, qui va interpréter “Tommy" à merveille, jusqu'à ne plus savoir qui il est. Ce livre ne cesse de s'interroger sur les liens entre la fiction et le réel, sur la construction cinématographique et l'identification qu'elle produit. Et nous rappelle qu'“à cette date (novembre 1943), et déjà depuis longtemps, il n'y avait plus guère que ces prodigieux métèques pour oser défier les Allemands en plein Paris".

\section{Missak de Didier Daeninckx (Perrin)}

18 Journaliste à L'Humanité, Louis Dragère est chargé en 1955 par les pontes du Parti communiste de retracer le parcours de Missak Manouchian, pour préparer l'hommage qui doit lui être rendu onze ans après son exécution. Situé dans le Paris populaire du milieu des années 40 et 50, le roman, mi-polar, mi-document, se présente comme une enquête auprès de différents témoins de la vie de ce résistant FTP-MOI (Qui a dénoncé Missak, et pour quelles raisons ?). Côté arménien, nous remontons à son exil d'Arménie, mais Dragère va aussi fouiller dans les archives, interviewer des personnages clés du PC (Duclos, Aragon, Krasucki...) et découvrir les limites de la vérité autorisée par le PC.

\section{La Mer noire de Kéthévane Davrichewy (Sabine Wespieser)}

Tamouna va fêter ses 90 ans. Elle n'aura plus assez de souffle pour éteindre les bougies, les visites de ses filles et petites-filles la fatiguent un peu, quelle effervescence, alors qu'elle ne pense qu'à son amour d'adolescente, le seul, Tamaz : viendra-t-il, celui-là ? Le roman entrelace avec grâce le récit de cette journée et le retour vers le passé : les beaux étés avec les grands-parents laissés en Géorgie, l'arrivée en France à l'âge de 15 ans (son père faisait partie du gouvernement qui a proclamé l'indépendance de la Géorgie en mai 1918), les cousins, les mariages, la Deuxième Guerre mondiale... Que reste-t-il d'une vie quand on a tant vécu?

\section{Patries de Jean Fratoni (L'Harmattan)}

Dans des notations éclatées, Fratoni évoque son adolescence à Miliana, en Algérie : les paysages, les assassinats, le couvre-feu, le départ en juillet 1962, l'arrivée dans le sud de la France... Pas de recension plaintive et accusatrice chez ce pied-noir pour parler de cette époque qui correspond pour lui à l'éveil du corps et de l'esprit. Un voyage en Algérie réactive ses souvenirs et lui fait regretter de ne pas avoir mieux connu la culture autochtone. L'auteur met en perspective l'exil des pieds-noirs avec celui de bien d'autres émigrés, comme ses aïeux venus s'implanter en Algérie... 


\section{En direction du vent de Fawaz Hussain (Non Lieu)}

21 Après vingt-cinq ans d'exil, un Kurde devenu français apprend la mort de son père au pays mais ne peut se rendre à ses funérailles. L'âge, la cinquantaine, le chômage, les migraines, la solitude - tout devient alors plus pesant. Le narrateur se déplace dans la capitale, et c'est le Paris des immigrés et des déshérités que l'on découvre avec son regard : la flicesse antillaise, le vendeur de fruits pakistanais, les caissières chinoises... Obsédé par son père, il traverse une période d'hallucinations à la Suleiman, le réalisateur palestinien: des grenades achetées dans le métro deviennent des engins explosifs, une agence de l'ANPE un cabaret oriental. Ce n'est qu'après un bref retour dans son pays natal qu'il retrouvera une certaine sérénité à Paris.

\section{Tâche de ne pas devenir folle de Vanessa Schneider (Stock)}

La mort de sa grand-mère paternelle est l'occasion pour la narratrice de revenir sur la vie pleine de frasques et d'enfants de son aïeule, du moins avant qu'elle soit enfermée dans une maison de retraite. L'évocation de la vie de Marthe, alias Ohé, passe par celle de ses parents, arrivés de Roumanie en France au début du XX ${ }^{\mathrm{e}}$ siècle. D'abord, il y a la réussite de son père, médecin à Bucarest avant d'être chercheur à l'Institut Pasteur, et qui retourne dans son pays comme soldat lors de la Première Guerre mondiale. On traverse aussi la Deuxième Guerre aux côtés de Marthe, de plus en plus borderline et pochtronne. Rien du roman narcissique dans cette saga d'une famille immigrée, car son histoire est aussi celle de tout le $\mathrm{XX}^{\mathrm{e}}$ siècle. La preuve, la narratrice se précipitera en Roumanie en 1989 après la chute du communisme.

\section{Ru Kim Thúy (Liana Levi)}

À travers de courts éclats de texte comme autant de fragments de mémoire, sans souci de chronologie, l'auteur revisite son enfance à Saigon (son père était préfet) et, après l'offensive du Têt en janvier 1968, l'année de ses 10 ans, la fuite dans la peur, le camp de réfugiés en Malaisie et la découverte d'un autre monde, le Québec. Par de multiples glissements entre le présent et le passé, Kim Thúy explore les traces laissées en elle par l'un et l'autre pays en alternant brefs récits et évocations sensorielles. D'une page à l'autre, on découvre à la fois une personnalité singulière et une expérience collective, une histoire intime et celle du Viêt-Nam, Une introspection pleine de sinuosités qui manifeste une grande soif de vivre et donne une leçon d'optimisme.

\section{Jusque dans nos bras d'Alice Zeniter (Albin Michel)}

24 Alice va se marier avec son copain d'enfance, Mad, pour qu'il ne soit pas expulsé vers le Mali. Une belle preuve d'amitié, et un nouveau chapitre de "la grande histoire du racisme", à laquelle travaille Alice, de père algérien et de mère normande (comme l'auteur), depuis l'époque du bac à sable où elle s'est fait traiter de "bougnoule". Ce roman très contemporain dans son inspiration comme dans son style et ses références ne cède pas aux clichés: si les jeunes gens se sont préparés avec angoisse au questionnaire destiné à déceler les mariages blancs, l'enquêtrice, qui n'a rien d'une 
inquisitrice, souhaite à ce joli couple tout le bonheur du monde. Malice et humour dans le regard sur soi, on échappe au pathos et aux bons sentiments.

\section{NOTES}

1. Palais de la Porte Dorée, 272, avenue Daumesnil, 75012 Paris. Métro ligne 8 : Porte Dorée, bus 46 ou PC2 : arrêt Porte Dorée.

2. Nouvelles Odyssées, éditions $\mathrm{CNHI}, 10$ euros, en vente à la librairie de la cité ou à commander sur le site : http://www.histoire-immigration.fr/ 